\title{
[ 452]
}

IV.

Bemerkungen über Wiesbadens Heilquellen;

ron

LEOPOLD GMELIN.

(Vorgelefen In der Gefellfchaft für Naturwiffenfchaft und Medlcin zu Heldelberg am 31 . Julius 1825.)

Wahrend meines Aufenthaltes zu Wiesbaden, im Sommer 1825, hatte ich Gelegenheit, über das dortige warme Mineralwafler einige Verfuche anzuftellen, welche zu folgenden Bemerknngen Veranlaflung gegeben liaben.

1) Ueber dle Wărme des Wlesbadener Wafrers.

Nicht blofs in Deutfchland, fondern eich in Frankreich *) herrfcht die Meinung, dafs die warmen Quellen ilıre überfchüffige Würme langfamer an das unigebende kaltere Medium abgeben, als irgend ein künftlich erwärmtes $W a f(e r$, diefes fey nun reines Wafter, oder durch Zufatz von Salzen dem Mineralwafer in feiner Mifchung ähnlich gemachtes Waffer, oder fogar das natürliche warme Waffer felbft, zuvor erkultet und dann künfliclı wieder erwärmt.

Dafs diefe wunderbare Eigenfchaft des langfameren Erkaltens auch dem warmen Wafer zu Wiesbaden zukomme, ift nicht blofs feit langer Zeit ent weder auf $T$ weu

*) Vergleiche die von Long champ in Aunales de Chimie et Physique T. 24. p. 248 bis 250 angefuhrten Behauptungen verfchiedener franzófifcher Aerzte. 


\section{[ 452$]$}

und Glauben, oder nach oberflählichen Beobachtungen angenommen, fondern es foll auch durch genanere Verfuche des Herrn Hofrath und Profeflor Kastner vollkonmen bewiefen worden feyn.

Die liefultate feiner Unterfuchnngen finden fich in: Wiesbaden und deffen Heilquellen, won Dr. Rullmann. Wiesbaden 1823. Sie find lant Vorrede S. xi vom Herrn Hofrath Kastner felbft dem Dr. Rullmann mitgetheilt worden,

S. 120 bis 123 diefes Werkes lieifst es:

„Kastner ftellte fowolil über dicfes langfame Er„kalten als über das eigentliche Gcleitetwerden der "Warme unfers Wafters mannichfache Verfuche an; „,unter andern auch nach einem Verfaliren, welches "dem des Dr. Trail nachgebildet war, vergl. Kast„ners Experinentalplyyfik, ste Auf. Bd.II. S. $5 g^{\circ}$, und "ernic'f folgende, fint cen Nathrforloher wic für den „ansübcrden Arzl höchlt merkwürdige Ergebnife:

„1) Das Waller unferer Quclle erkaltet, unter übri,gens genau gleichen Bedingungen, bedeutend "langfomer, als reines Walier mind als Salzwaller "von denfelben kigengewichte.

22) Die Lrkillungsdaucr ift grülier, als fie feyn foll"te, wemn fic, wio bei einer kïnfilichen Salzlö"fiung, im zulammengefetzten verkelirten Ver„hältuils der Wörmeleitung *) und Würneftralı-

*) Herr Hofrath Kastner hat demnach die Wirmeleitung von den im Wiesbatener Mineralwafter enthaltenen Salzen nach ihrer Auflofung in Waffer geprifr; es ift zu wünfchen, dafs fie dereinft bekannt gemacht werden, da nur hierdurch dem Refiltate foiner Bereclinung ein gröfseres Zutrauen verfchaffl werden kann. Gra. 


\section{$\left[\begin{array}{ll}453 & \text { ] }\end{array}\right.$}

„lung "), und im zufammengefetzten geraden der "Zähigkeit (Cohafion) und der chemilchen Angeieluung (Bindungsftärke) zwifchen dem Waffer „und den darin gelölten Subftanzen *) ftände.

„Ueber die Verfahrungsweife, nach welcher „Ka st n er diefe mitunter fehr delicaten Verfuche an„ffellte, wie er das Maafs der Wärmeleitung und „Wurmeftrahlung, das der Zäligkeit und der dielel„be bedingenden Trübheit, und das der chemifchen "Anzielınng des Löfungemittełs zu den nelöften Sub„ftanzen erforlchte, verweife ich die Lefer, die Inter"effe dafür haben, bis Kast ner lelbft etwas darüber „,bekannt macht; anf dellen Experimentalphyl: ste Aufl. I. Bd. 1-3tes, und II. Bd. 7. 30 and 11tes Cap. ${ }^{* * *}$ ).

-) Vor der Hand ift es mir nicht erklirlich, In wiefern die Wärmettrahlung hierbei in Betracht kommt, da fich die, in Gefilse yon gleicher Natur eingefchloffenen, Flüffigkeiten hinfichtlich der Wirme-Ausfrahlung gleich verhalten müfen. $\mathbf{G m}$.

*) Aach die Berïckficlatigung diefes Moments ift mir nicht ganz verftindlich. Herr Hofrath $\mathrm{K}$ a stner wird aufser mir gewifs noch viele andre Lefer verbinden, wenn er erklart, in wiefern die chemifche Anziehung zwifchen dem Waffer und den darin gelöften Subftanzen Einflufs auf das fchnellere oder langSamere Entwejchen der Wärme aus der Lofung hat. Oder follte der Erkätungsverfuch in einein Gelafse mit weiter Mündung angeftellt worden feyn, wobei die Erkhltung durch Aasfrahlung aus der Flitfigkeit nach oben und durch Dampfbildung (die boi reinem Walfer bedeutender feyn inufs als bel falzhaltigem) mit in Anfchlag kame? Doch dann liefse fich gegen die $Z$ weckmafsigkeit des Verfuchs Manches einwenden.

Gm.

***) Vielleicht würde demjenigen, dor, wie verlangt wird, alle 


\section{[ 4554 ]}

"Die bedeutend gröfsere Erkaltungsdauer, wel"che unlere Mineralwăler in Kastners Verfuchen "darboten, lief's denfelben eine verhältnilsmäfsig gro"lise Menge gebundener Wärme darin vermuthen, "wiewolll er aus anderweitigen Verfuchen folgert, dals "anch die trübende, und die Zühigkeit des Waflers „erhöhende Subftanz (dio Kiefelthonerdo fanmt dem "Extract) an dielem auffallend lang famen Erkalten „beträchilichen Antheil habe. Un dio Menge der ge„bundenen Wärme auszumitteln, komnto das Erkal„ten lelbft nicht gewählt werden, da auf dallelbe (wie "ebcn bemerkt wurde) (ehr verfchiedene Urfachen in"fluiren. Kastner war dalıer genöthigt, andere „Wego aufzuliuchen, welche, möglichft frei gelaflen „von jenen Influenzen, ficherer zum Ziele fülurten; er "erprobte demnach

"1) die Capacität unferes, vorher vorfichtig erka'"teten, Wallers für die Wärme, durch Zumi„'chung von reinem Waffer, unter felır mannich-

diefe Capitel durchlief, Aufklărung zuflefsen. Alleln diefo Capitel, welche die Lehre von der Schwere, vom Drucke der Flüfigen, von der Anhaftung und Zurammenhaltung der Körper, von dem chemifchen Proceffe, von dem Lichto und von der Wàrme enthalten, arachen nicht weniger als 205 Seiten, d. h. melir als die Hilfte des ganzen Werkes ans. Einer folchen Zunuthung habe ich unmöglich Genüge leiften können; lieber will ich meine Unkenutnifs geftehen, und Herrn Hofrath $k$ astuer bitten, entweder die eimzelnen Stellen feines Werks zu citiren, weiche zu meiner und der ibrigen Lefer Belehrung dienen könuen, oder was noch genügender feyn wirde, reine Beobazhtungen über Warmeleitung u. fo w. und die Art, wie er diefelbe der Bereclunng unterworien hat, verfprochenermafsen austührlich bekannt zu macien. 


\section{[ 455 ]}

„fach abgelnderten Wiederholungen; ftets war "die Temperatur des Gemifclees geringer, als fie „hattte feyn müffen, wenn reines $\mathrm{W}$ afler mit rei"nem Waffer, oder mit künftlichen Salzlöfungen "vorinifcht worden wäre;

"2) beftimmte er die Menge Eis, welcho durch be„fimmte Quantitäten unferes Waffers sefclimol"zen wurden, und jedesmal übertraf diefe Mengé „diejenige, welche reines, oder künftlich falzlıal„tiges, eben fo heifses, Waffcr zum Sclimelzen „brachte, um ein Bedentendes.

„Hiernach enthält unfer Thermalwafer an fich „mehr gebundene tö ürme als reines $\mathrm{W}$ a fler, oder als „ein, dem liefigen ähnliches, Kunftgemilch, wnd "führt daher dem menfchlichen Körper, der es im „Bade in fo bedeutender Menge einfaugt, auch ver"haaltnilsmäfsig mehr gebundene Wärme su, wälh„rend es leine fülllbare $W$ ärme in längerer Zeit mit"theilt *).6

-) Die belden unter $I$ und 2 angefuhrten Verfuche wirden, thre Richtigkels vorausgefetzt, nichts anderes beweifen, als dafs das natürliche Mineralwaffer cine grobfsere fpecifiche Wärme hat, als reines oder klinfltch nachgeahmtes Mineralwaffer, d. h. dafs es mehr froior $W$ ärmo bedarf, um bis auf eine gewiffe höhere Temperatur zu kominen, und dafs es bei der Erkältung um elne gewiffe Zabl von Graden eine grölsero Menge von froier, ungobundonor Wärmo entlafst. Wio aber ein Phyfiker (welcher in Seiner fo oft citirten Experimentalphyfik B. 2. S. 565 fagt: "Man nimmi nämlich an, dafs fich der „Warmeftoff mit andern Stoffell fo verbiuden könne, dafs er, "an feiner Verbreitung vollkommen gehindert, auf keine Uin"gebung ausdehnend zu wirken vermoge und fomit auch nicht 


\section{$\left[\begin{array}{ll}456 & 1\end{array}\right.$}

Die Thatfache des langfameren Erkaliens eines warmen Mineralwaffers als riclutig angenommen, fo mufs fie liegen: entweder I. darin, dafs man dafelbo leinen wigbaren Beftandtheilen nach niclıt gehörig kennt, urd allo anch kein künfiliches Wafler von derfelben Befchaffenheit lhervorzubringen vermag; oder II. dafs uns bei der chemifchen Unterfuchung des Wâfers unwägbare, anf das Ausftrömen der Wärme einflielsende, Beftandtheile entgehen; oder III. dafe die Wärme der Mineralwatfer eine andere Natur hat, als künftlich in Waffer gebrachte Wärme; oder IV. dals lie auf andere YYeife mit dem Wafter verbunden ift.

I. Dic Analyfe hinfichtlich der wigbaren Beftandtheile kann mangelhaft (iyn, theils 1) in der Beftimmung derfelben an und fitr fich, theils 2) in der Beftimmung ilher Verbindungsweile.

1) Nienand, der einigermalsen mit den Schwierigkeiten der Analyfe von Mineralwaflern bekannt ift,

„empfunden werden künne; and nennt ihn dann verborgenen "oder ruhendon, $b$ und.on on oder latenton War„meitolf, im Gegenfatz des fühlbarin, fenfibeln oder freion "W'ärmeftolfs, welcher Temperaturerhühung der Körper her„vorbringt und auf unfer Gefühl und auf das Thermometer "wirkt." - Und S. 572: „Hingegen nemut man die [nicht abfolut $z u$ beltimmende] "Würmemonge, welche jede eigen,thtimliche Materie [bei beftinmter Maffe] nöthig hat, um "dadurch eine beftinmte Temperatur zu erreichen, ihre Jpo"cififche, comparativo, eigenthümlicho Wiarmo)" - wio diefer Phyfiker aus diefen Verfuchen auf eine grobfsere Mengo von gebundener Wrärme im Wiesbadener Waffer sclsliefsen kann, it ein Kithfel, deffen Lüfung doch deın Herrn Hofrath Kastaer am meiften am Herzen liegen folite. Gm. 


\section{[ 457 ]}

wird lăngnen, dafs hierbei einzelne Beftandtheile niberfehen, eine Materie für die andere gehalten und das Gewicht der einzelnen Beftandtheile unrichtig beftimmt werden könne. Die anffallenden Entdeckungen von Stoffen in den Mineralwaflern durch Berzeli us, die man nicht darin ahnete, die fo abweichenden Ergebniffe der über ein und daffelbe Mineral wafer durch verfchiedene Chemiker angeftellten Analyfen beweifen diefs linulanglich.

Um jedoch die Wiclıtigkeit eines folchen Verfelhens hinfichtlich der Erkalltungsdaner beurtheilen zu können, wollen wir Kirwans Erfahrung über die fpecififche WVärme der Kochfalzauflöfung zu Grunde legen. Eine Auflöfung von 1 Theil Kochfalz in $8 \mathrm{Thl}$. Wafter hat nach ilun eine lpecifilche Wärme oder Wärmecapacitat bei gleichen Gewichten von o,8jz, die des Wafers $=1$ geletzt. Hicrnacl würde die relative Würme diefer Kochfalzlöfung, oder ihre Wärmecapacitat bei gleichen Umfang, da das fpecififche Gewicht derlelben nach Watson 1,074 beträgt, $0,832 \cdot 1,074=0,393568$ feyn, oder etwas über to weniger betragen, als die des reinen Vaflers. 1 Pfund Wiesbadener Wafier' entlıält nach einem Durchlchnitt von Kastners und Lades Verfuchen 6o Gran fefte Theile. Wir wollen diefe für Kochfalz nehmen, da dieles wenigftens bei weitem die Hauptmafe ausmacht, und da ohne Zweifel die übrigen falzigen Befrandtheile, dem Kochfalz uhnlich, die Warmecapacitat des Waffers vermindern. Da 2 Pfund 128 Draclimen beträgt, und 6o Gran 1 Draclime machen, fo hătten wir hier eine Auflöfung von 1 Theil Kochfalz in 127 Theilen Walter. Wir können diefes Wafler als ein Gemifch 


\section{[ 458 ]}

betrachten vin 119 Theilen reinen Waffers und von 9 Theilen einer Auflöfung von 1 Theil Kochfalz in 8 Theilen Waffer, und annehmen, dafs fich hieraus die V ärmecapacitalt diefer felır verdünnten Kochfalzlölung ziemlich richtig werde berechnen laften. Die fpecififclie Warme des Mineralwaffers wăre hiernach $=\frac{9 \cdot 0,832+119 \cdot 1,000}{128}=0,988$, und die relative Würme wäre $\frac{\left.8,38 \cdot 0,893568^{\circ}\right)+119 \cdot 1,000}{127,38}=0,99^{3}$; oder, da Kastner das Ppecififclie Gewiclit des Wiesbadener Waflers $=1,006$ gefunden liat, lo ergiebt fich lieraus die relative Wärme $=0,988 \cdot 1,006=0,993928$, was mit der erften Berechnungsart lo gut wie ganz übereinftinumt.

Aus diefen Berechnungen ergiebt fich folgendes Refultat: Es giebt, foviel bekannt (wovon blofs nach $\mathrm{Dalton}$, keinesweges aber nach $\mathrm{Kir}$ an das einzige wallrige Ammoniak auggenommen werden mufs), keine fefte oder tropfbar flüffige Materie, deren Ipecififche Wärme der des Waffers gleich käme. Verbindungen diefer Subftanzen mit Wafler befitzen daher, wie es fich in Voraus vermuthen lälst, und wie es anch die Erfahrung bis dahin bewăhrt liat, eine geringere fpecifilche Wärme, als das reine Wafter. Diefes ift daher auch der Fall mit fammtlichen Mineralwafiern. Jedoch ift die Menge der in ihnen aufgelöften fremdartigen Stoffe meift fo unbedeutend, dafs hierdurch

* Sofern 9 Gewichtstheile Kochfalzl5fung, deren fpecififches Gewicht $=1,074$ und deren specificte Warme $=0,832$ (wenn man Gewicht und Mars bei Waffer $=1$ fetzt) Eleich linul 8,38 Maafsen vou $0,893 j 68$ relativer Wärme. 


\section{$1459]$}

die fpeciffele Warme des Waffers nnr um ein Geringes vermindert wird. Vergleichen wir endlich folclro Mineralwafler mit dem roinen Walfer nicht dem Gewicht nach, fondern dem Maafse nach, fo zeigt fich in ihrer Warmecapacităt ein höcluft geringer Unterfchied, namentlich zcigt fich die relative Wärme des Wiesbadener Waflers, welches unter die reichhaltigeren Mineralwafler gehört, $=0,993$, und unterfuchen wir direct, ob roines Walfer oder Mineralwaller, welche beide zu gleichen Maafsen gegeben find, fchneller erkaltet, fo wird fich kein auffallender Unterfclied darbieten.

Mögen daher den bisherigen Analyfen eines Mineralwalters 10 befondere Stoffe entgangen leyn; mögen diejenigen, welche lieber ahnen als forfchen, noch 100 unbekannte Wefen darin annelimen, fo kann, fo lange man noch keinen Stoff kennt, von welchem eine Kleinigkeit die fpecifiche IVarme des Wafters bedeutend erhölıt, und lo lange wir annehmen müfen; dafo alle vom Wafler gelöfte Körper feine Capacität bald etwas melir, bald etwas weniger vernindern, keine Aenderung in der Hauplfache eintreten, um fo mehr, als, wenn einmal das Totalgewicht der gelöften Körper beftimnt ift, im Verhältnifs des Gewichles der etwa unentdeckt gebliebenen Stofle das Gewicht der aufgefundenen verringert angenommen werden mufs. Kurz alle Mineralwalfer werden eine geringere fpecififohe $\mathrm{V}^{\mathrm{r}}$ arme befizen, als reines Wafler; da fie jedoch meiftens wenig gelöft enthalten, fo wird diefer Unterlchied gering leyn, und noch geringer der Unterfchicd zwilchen der relativen Wärme des reinen 


\section{[ 460 ]}

Wafters und des Mineralwaflers feyn, weil letzteres Ppecififch lichwerer ift.

2) Dic chemifcho Analyfe könnte auch in fofern fehlen, als fie zwar die Beftandiheile richtig erkennt, aber nicht die Art, nach welcher diefelben verbunden find. $8 \mathrm{chweigger}$ erwahnt in feinem Journal Bd. 3g. S. 386 einiger mit $R$ eu $\int s$ und Seiler angefellter Verfuche, nach welchen es ilhm fchien, daf's natürlich warmes, und nach dem Erkalten wieder erwämtes Teplitzer Walfer zwar von $37^{\circ}$ bis $24^{\circ} \mathrm{R}$. gleich fclinell crkalteten, dann aber, bei niederen Temperaturgraden, erftercs längere Zeit warm blieb. Ol.ne diefo Verfuche als abgemacht zu betrachten (das Refultat der wcitern Unterfuchungen, welche hierüber Reul's anzuftellen gedachte, ift meines Wiflens noch nicht bekannt gemacht) fucht Schweigger diefe Thatfache vermuthungsweile aus der Annalime zan erkluren, dals in warmen Mineralwafern die Beftandtheile der Salze mehr zu einém gemeinfclıaflichen Ganzen verbunden find, und erft bei einer gewillen niedrigen Temperatur zu den nehr binären Salzen zufammentreten, wie fio der Chemiker findet; dals endlich hierbei Waimeentwicklung Statt finde.

Diefe Erklärung würde noch die walufcheinlichfte Zufluchı für den Fall bleiben, dal's wirklich bei einigen warmen IVaftern ein langfameres Erlaalten bewicfen würdc. Nur könnte man einwenden: Wenn fich im Teplitzer Váfer erft unter 24 "R. die einzelnen Salze erzengen, fo mufs über dieler Temperatur die Kraft, welche das gemeinfchaftliche Salz zufammenlualt, vorgeherrfcht habcn; dann mufo aber anch im erkalteten Waffer bei feinem Erwürmen über $24^{\circ}$ 


\section{[ 461$]$}

dje Bildung des gemeinfchaftlichein Salzes nus den einzelnen Salzen wiederum bewirkt worden feyn, Hier auf liefse fich zwar antworten: die in Salzfäure gelölt gewefene Kiefelerde zeigt fich nach dem Abdampfen unauflöslich in Salzfäure, auch wenn diefelben $\mathrm{Um}$. fiande eintreten, als unter denen fie gelöft gewefen war, und ahnliche Fälle. In allen diefon ift aber eino fefte Subftanz und dio noch niclıt genügend erklärte Wirkung der Cohäfion gegeben, ein Umftand, der bei einer wâfrigen Flülligkeit, in welcher fämmtlicho Theile gelöft bleiben, wegfüllt. - Auch könnte man nach der Cchon öfters beliebten Weile ein Mineralwafler als eine organilche Verbindung anfehen, die ficlı zwar decomponiren, aber niclit wieder componiren laffe. Doch ift hierzn zu wenig Grund vorhanden, als dafs es fich der Mühe lohnt, diefe Anficht hier zu beftreiten.

- 11. Was nun die Imponderabilien betrifft, die man in den natürlichen Mincralwafern vermuthet, fo können dieles theils bekannte, theils unbekannte feyn: Von den bekannten wird vorzüglich die Elektricität genannt. Die (von VValcker bereits beleuchteten) Verfuche jedoch, welche nach Kastner deren Gegena wart in den Mineralwaliern beweifen follen (S. 123 des Rullmann (chen W $W$ erkes), foheinen mir wenigftens etwas ganz anderes zu beweilen. Wáren dicfo Vera fuche und die daraus gezogenen Schlüffe richtig, fo würde fich behaupten lafen, verdünnte Sclıwefelfäure fey noch viel reicher an logenannter Mifshungselek. tricitat, und eine folche Flïlfigkeit müfo (wenigftens in der Art der medicinilichen Wirkung, die man von

Aunal. d. Phyfiks B. 83، St. 4t J. 1826 s S: 6 , 


\section{$\left[\begin{array}{ll}46_{2} & ]\end{array}\right]$}

der Mifchungselektricität abgeleilct wiffen will) dio natürlichen Mineralwailer weit übertreffen.

Noch weniger läfst fich von den unbekannten Imponderabilien fagen, welche Kastner in f. Archiv Band 1. Heft 2, in den Mineralwaftern anzunelimen geneigt ift, und zu welchen anch wohl der von Rullmann (Vorrede S. xuI) citirte Brunnengeift zu rechnon ift. Ihre Annahme gründet fich lediglich anf dio Erfalirung, dafs fich melirere Mincralwafter heilfari zeigen, wie Schlangenbad und Pfeffers; in denen der Chemiker aufser dem Wâfer faft nichts Ponderabeles gefunden habe. Allein von der Möglichkeit abgefeJien, dafs irgend et was Ponderabeles in diefen Waffern, welches fchon in kleiner Menge fehr wirkfam ift (ich erinnere nur an Jod) unbeachtet blieb; fo weifs man, wie fchwierig es ift, die Heilung einer Krankleit als Wirkung des angewandten Heilmittels mit Sicherheit zu betrachten, da fich die Natur oft felbft hilft, befonders wenn das Heilmittel Wochen hindurch gebraucht werden mufs, und da es unmöglich ift, mit Gewifsheit anzugeben, dafs der Gebranch des gewöhnlichien Quelliwafters bei derlelben Lebensweil'e n. f. w. nicht gerade diefelbe Wirkung würdo hervorgebracht haben.

Lafst fich demnach die Gegenwart bekannter oder unbekannter Imponderabilien (aufser der VVärme) in den Mineralquellen nicht gerade widerlegen, fo ift fio noch viel weniger erwielen und möchte vor der IIand ftark zu bezweifeln feyn.

III. Die Annahme, die Mineralwater enthielten eine andere Art von Würme, als diejenige, die wir 


\section{$\left[\begin{array}{lll}463 & \end{array}\right]$}

künfllich zu erzengen vermögen, bedarf koines VViderlegung.

IV. Endlich bleibt noch übrig, die angeblich janglamere Erkaltung des Mineralwallers von der Art; wie die Würme mit dem Mineralwafler verbunden ift; abzuleiten. Hier liffst fich aber fagen: die in irgend einem Körper enthaltene Wurme ift theils freie, theils gebundene. Vou der letztern kann hicr nicht die Re-

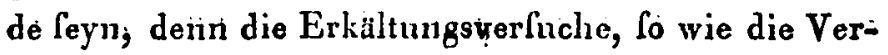
fuche, in welchen Eis gelchmolzen wird; befchaftigent fich blofs mist der freien.

Es fragt fich jetzt, ob es mit den Begriffen; welche wir von der freien Würme haben, vereinbar ift; wenn wir annehmen, zwei Flüffigkeiten; welche ihren ponderabeln Theilen nach ganż gleich find; und worin fich keine Verfchiedenlieit hinfichtlich unwagbarer Stoffè nachweifen läst (nämlich das natürlichò und künftliche Mineralwa(ter), könnten die freie Wurrme iit einem verlchieden freien Znftande enthalten; fo dafs diefelbe zwar ans beiden Flülfigkeiten entweicht, ans der einen aber fchneller als ans der an: dern:

Für jetzt glaube ich mit Nein antworten zu müffen. Denn die Plyfik lehrt fonft nichts der gleichen, und es liefse fich von dem Grand einer folclien Verfchiedenlieit nicht wohl Rechenfchaft geben: Möge die Warme dem natürlichen Mineral wa afer auch durch die grörste Hitze vulkanifchen Feners und uniter deni gewaltigften Drucke im Innern der Erde nitgetheilt worden feyn, wälırend das künfliche allimalig und mäfsig erwämt wurde. Ift erfteres wieder auf denfelben Punkt erkaltet, bis zn welchem letzteres er- 


\section{$\left[\begin{array}{ll}464 & \text { ] }\end{array}\right.$}

warmt wurde, fo worden beide von da an gleich viol freie Wärme enthalten und gleich \{clinelb erkalten müßen.

Bei folchen Zweifeln gegen die allgemein herrt fchende Meinung glanbte ich bei meiner Anwefenheit in Wiesbaden die Erkalıngsverfuche wiederholen zu mïfen. Ich that diefes in Verbindung mit Herrn Hofapotheker $\mathrm{L}$ a de, demfelben, der f́chon vielfältige Unterfuchungen tiber die Wiesbadener Quellen angeftellt hat.

Wir wählten zu unfern Verluchen 4 gewöhnliche grüne cylindrifclie, ungefahr 8 Unzen Wafter haltende, Medicinglafer von möglich ft gleicher Form und Dicke und von gleichem Inhalte. Wir bereiteten künftliches Mineralwafer, indem wir uns hierbei an die Analyfe Kas Iners hielten, und löften zu dicfom Behnf in 4 Pfund Waffer anf: 200,00 Gran getrock* netes Kochfalz, 4,25 Gr. kryftallifirtes Glauberfalz, 2,12 Gr. kryftallifirten Gyps, 21,56 Gr. Chlorcalcium und 2,8 Gr. in der genan erforderliohen Menge von Salzfäuro gelöfte Bittererde *). Das Gemifch hatte einen dem natürlichen Waffer ähnlichen Gefchmack. Wir füllten bis an den Hals das eine diefer Glafer mit deftillirtem Waffer, ein zweites mit dem künflichen Mineralwaffer, ein drittes mit natürlichem Mineralwaffer, welches wir an der Luft bis auf $18^{\circ} \mathrm{R}$. liatten

-) Vom Kochfalz haben wir einige Gran mehr genommen, als es zufolge der Analyfe von K a st ner hätte feyn follen; I Pfund Mineralwaffer giebt nimlich nach $K$ astners Analyfe befinaho 58 Gran fefte Theile, nach $L$ a d e's Verfuchen 62 ; wir baben das Mittel genommen. 


\section{$\left[\begin{array}{lll}45 \tilde{j}] & {[}\end{array}\right.$}

erkalten daffen. Diefo 3 Gläler wurden jetzt, neben einander an Bindfüden aufgehängt, $\frac{1}{2}$ Stunde lang bis an den Hals in die Quelle des Kochibrunnens gelenkt, lo dad's ihr Inlhalt dio Temperatur dieler Quelle annchmen mulste. Hierauf fillhen wir anch das vierte leergebliebene, aber durch Schwimmen auf dem Koclibrumen erwärmle Glas mit dem leifsen Wafter diefes Brunnens, fo dafs fich in jedem Glafe ein gleiches Maafs Flüfligkeit befand. Damn loben wir alle 4 Gläfer faft gleichzeilig aus dem brunnen, trockneten fio fclnell mit einem Leintuch ab, ftellten fie auf ein Jjet und brachten fie fo in ein 10 Schritt davon entferntes Kans. Die Temperatur beftinmenten wir jedesmal, nachdem wir zuerfit nach dem Verfchliefsen der Oeffnung mit einem lïnger die warnere und kältere Sclicht durch Schütteln gemengt hatten, mittelft eines fehr kleinen Thermometers, welches wir ab?wechfelnd in einem diefer Gläfer fiehen lielsen, damit es immer ungefalır diefelbe Temperatur behielt, und nicht etwa, nach dem Erkalt'n in dar Luft, der Hiäffigkeit, in welche es zuerfi getauclit wurde, Wärne entziehen könne.

Lndlich wechfulten wir bei einem zweiten Verfuche die Gläfer, die wir mit $A, B, C, D$ bezeichnen wollcn, um uns zu überzengen, dul's nicht ctwa verfchiec'one Dicke und Oberflächo der Gläler einen bedeuleuden Einilul's auf das lịefinltat geliabt habẹ. 


\section{[ 466 ]}

Verfuch 1. Temperatur des Zinmers $18^{\circ} \mathrm{R}$.

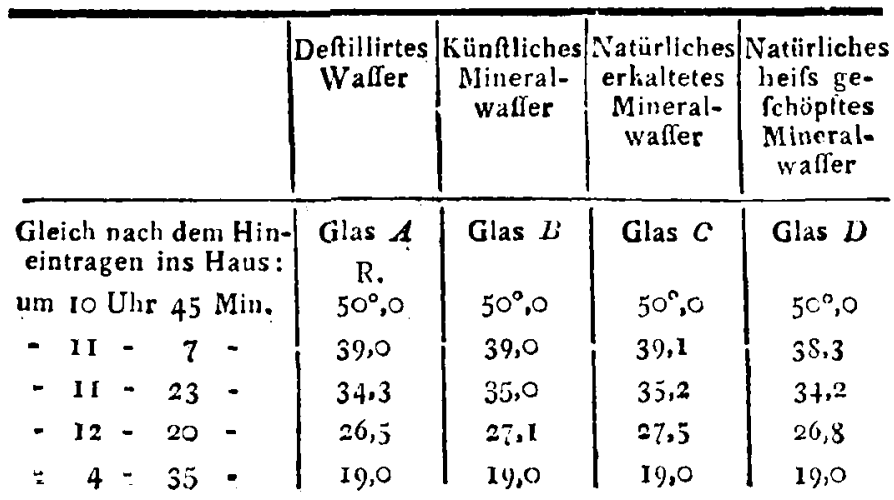

Verfuch 2. Temperatur des Zimmers $19^{\circ} \mathrm{ll}$.

\begin{tabular}{|c|c|c|c|c|}
\hline & $\begin{array}{c}\text { Deftillirtes } \\
\text { Waller }\end{array}$ & $\begin{array}{c}\text { Künftliches } \\
\text { Mineral- } \\
\text { waffep }\end{array}$ & $\begin{array}{c}\text { Erkaltetes } \\
\text { natlirliches } \\
\text { Mineral. } \\
\text { walfer }\end{array}$ & $\begin{array}{l}\text { Heifs ge- } \\
\text { fcliöpftes } \\
\text { natürliclıes } \\
\text { Mineral- } \\
\text { waffer }\end{array}$ \\
\hline $\begin{array}{l}\text { Gleich nach dem Hin- } \\
\text { eintragen ins Haus: }\end{array}$ & $\underset{\mathrm{R} .}{\mathrm{Glas}} C$ & Glas $D$ & Glas $A$ & Glas $B$ \\
\hline um 5 Uhr 30 Min. & $50^{\circ}, 0$ & $50^{n}, 0$ & $50^{\prime \prime}, 0$ & $50^{\circ}, 0$ \\
\hline$-6 \cdot 10=$ & 37.7 & 37,8 & 36,5 & 37.5 \\
\hline$=7.15$. & 25,7 & $26, I$ & 25,0 & 26,1 \\
\hline
\end{tabular}

Ein dritter Verfucl, bei welchem wir blofs das künftliche mit dem heifsgefchüpften natürlichen Miņeralwafier vergliclien, ohne jedoch die Zeiten unferer Beobachtungen genau zu bemerken, gab dalfelbo Refultat, mämlich dafs alle diefe Flülfigkeiten ungefälır in derfelben Zeit erkalteten, und dafs die kleinen Yerfcliedenheiten in der gefundenen Temperatur wohl nur von Beobachtungsfehlern herrülırẹ, z. B. yon nicht hinreichendem Schülteln vor dem Fintau- 


\section{$\left[\begin{array}{lll}{[} & 457\end{array}\right]$}

clien des Thermomelers, divon, dufs die Beobachitung nicht bei allen 4 lilülfinkciten zugleich angeliellt werden konnte, londern cinn nach der andern, wobei dio Cpäter unterfucliten fich elwas kảler zeigen komnten, dals das Glas $A$, wie es licheint, otwas fichneller dio Wärme angfralılte u, l:w. Wie diele Verfuche, welche zu demfelben Relinltat fïlnen, wclches Longchamp *) crlalten laut, unit den von Kastner zu rcinen feyn mögen, bin ich nicht in Stande zu beurtheilen. Doch wird das verfchiedene Refultat unferer Verliche noch anclere Naturforfcher auffordern, durch nene Verfuche cine Entfcheidung lierbeizufül ren. Es wird, wie ich holle, duzu beitragen, den magifchen Sclileier, in welchen man to gern die Ileilquellen und dic Urlache ihrer Wirkfamkcit hüllt, in dein Nichits zerflief'sen zu machen.

2) Ueber das fich aus dem Kochbrunnen in Blafen entwirkelnde Gas.

Diefes Gas ift hereits von Lade und von Kastner mulerficht worden. lirfterer find darin zufolge einer mir mündlich gennchten Nithleilung 85,5 $\mathrm{pl}$. kohlculaures Gas und $16,7, \mathrm{C}$. Stickgas, in welcliem er noch clwas Saucribiligus vermuthele. leetzterer ( $f$. Rullmanns Wiesbaden S. 14it) fand dicles Gas aus 54 pl. kohlenlaurem Cale und af plo. Stickgas zulanmengaletzt. Die Abweichung diefer beiden Refultate veranlafite mich, in Verbindung mit Herrn Lade den Verfinclı an wiederlıolen.

Vir finden, dul's dis Unfangsvermindernng, welche hali in dem über (Gucckfilber befindlichen Gale

-) Aunales do Chinie et Pliysique. T. 24. p. 247. 


\section{[ 468 ]}

liervorbrachto (nach der nötligen Correction für Tenıperatur und verfchiedene Höhe der Quechfilberfäule) 82,3 l'rocent betrug, was mit dem früheren Refultate von Lade faft gänzlich übercinftimnt. Andererfeits fclıcint der nicht durch Kali ablorbirbare Theil reines Stickgas za leyn; wenigftens licfs fich bei $\frac{x}{4}$ ftündigenn Schütteln nit Schwefelleberlöfung kcine AbCorption bemerken,

3) Ueber elnigo Beftandthelle des Badfinters.

Zu den von Kastner in Wiesbadencr Wafter gefundenen Beftandtheilen (vergl. Mullmanns Werk, S. 150 n. 15ı) münen noch linzugefügt werden: Fllıor und Mangan. Wenigftens erlielt ich durch Auflöfen des Badfinters in Salzfäure und Fällen der filtrirten Löfung mit Ammoniak einen Niederfchlag, welcher, mit Schwefelfänre erhitzt, Flufsläure entwickelte *) und weleher, nach der Auflöfung in Salzfiurc und Abfcheidung des Lifens mittelft doppelt kohlenfauren Natrons, mit Kali cinen reichlichen Niederfclulag gab, der grölstentheils aus Manganoxyd beftand. Selbft der ganze badfinter gab unit Borax cin in der Kälte violett erfcheinendes Glas.

*) Icls pflege dio Glasplatte mlt einem Aetzgrund zu überziehen, der bei $1 \mathrm{CO}^{\circ}$ C. nicht fehmilat (z. B. aus gleichen Thcilen Asphalt, Schellack und Wachs zufammengefchmolzen), und den mit diefer Platte bedeckten Platintiegel, welcher das Gemifch enthät, einige Tage auf dem Wafferbado zu laffen. Auch dic von Bcrzelius empfoliene Vorficht, ein wciches Nietall zum Ritzen des Aetzgrupdes anzuwenden, wurde nicht vernachlorisigt. 